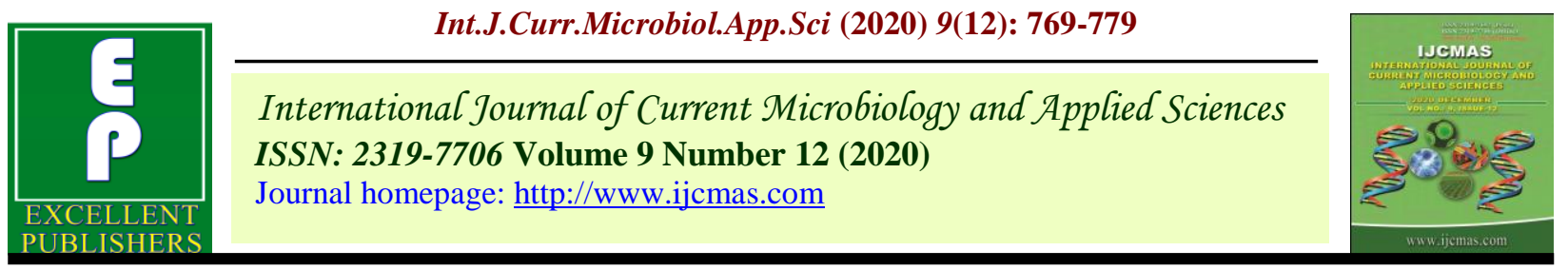

\title{
Effect of VA Mycorrhizal Fungus and Bioformulations on Quality, Root Parameters, Microbial Analysis and Economics of Fennel under Northern Dry Zone (ZONE-3) of Karnataka
}

\author{
Shashidhar M. Dodamani ${ }^{1 *}$, N. K. Hegde ${ }^{1}$, Chaya P. Patil ${ }^{1}$, \\ Laxman Kukanoor ${ }^{2}$, T. B. Alloli ${ }^{1}$ and Krishna Kurubetta ${ }^{3}$
}

${ }^{1}$ Department of Horticulture, Kittur Rani Channamma College of Horticulture, Arabhavi,

${ }^{2}$ Kumbapur Farm Dharwad, ${ }^{3}$ Agronomy, HRES, Devihosur, University of Horticultural

Sciences, Bagalkot, Karnataka, India

*Corresponding author

\begin{tabular}{|c|c|}
\hline & A B S T R A C T \\
\hline & \multirow{8}{*}{ 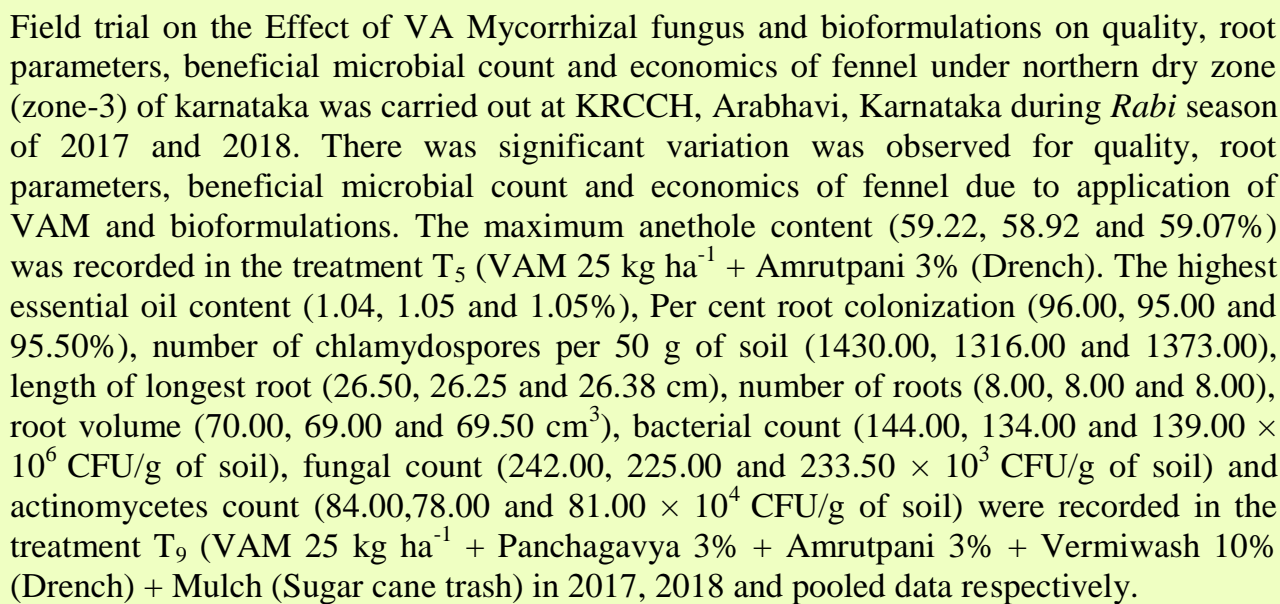 } \\
\hline Fennel, VA & \\
\hline $\begin{array}{l}\text { Mycorrhizal fungus, } \\
\text { Bioformulations, }\end{array}$ & \\
\hline Quality, Root & \\
\hline $\begin{array}{l}\text { parameters, } \\
\text { Beneficial microbial }\end{array}$ & \\
\hline count, Economics & \\
\hline Article Info & \\
\hline $\begin{array}{l}\text { Accepted: } \\
07 \text { November } 2020 \\
\text { Available Online: } \\
10 \text { December } 2020\end{array}$ & \\
\hline
\end{tabular}

\section{Introduction}

India is known as the land of spices from the times immemorial and has been the global leader in the production, consumption and export of spices. Fennel (Foeniculum vulgare Mill.) is one of the popular seed spice in India mainly grown in rabi season. It is locally known as saunf and belongs to the family
Apiaceae (Umbelliferae). Fennel is native to Southern Europe and Mediterranean region. It is a hardy perennial, but is grown as annual or biennial. It is cultivated throughout the temperate and subtropical regions of the world for its aromatic seeds which are used for culinary purpose. Fennel seed is small, oblong or cylindrical, 6-8 $\mathrm{mm}$. long straight or slightly curved yellowish brown. It 
possesses an agreeable, aromatic and sweet aroma due to higher content of volatile oil (0.7 to $1.2 \%)$.

The volatile oil contents mainly anethole $(50.03 \%)$ and fenchone (2.67\%). It is widely used as flavouring agent in culinary preparations, confectioneries, cordials and liquors. Fennel oil is also used as important ingredient in several allopathic as well as ayurvedic medicines which are used in diseases viz., diabetes, bronchitis and chronic coughs, treatment of kidney stones and is considered to have diuretic and galactogogue properties. The use of bio-fertilizers and bioformulations play an important role as they help in availability and supply of plant nutrients thereby, providing a scope for reduction in use of costly chemical fertilizers which pollute soil in long term (Kale et al., $1991)^{2}$. It is reported that 10 to 20 per cent of crop yield can be increased with biofertilizer application alone (Brown, 1972) ${ }^{1}$. The increasing concern about the environment and socio-economic impact of chemical agriculture has led to seek alternative practices for agricultural sustainability and marketability by progressive farmers. To minimize the adverse effects of conventional agriculture (e.g. polluted water and soil by chemical fertilizers, entering pesticides to the food chain, compaction of the soil by heavy machinery etc.), different alternative concepts of production have been developed. Currently, low input cropping systems and innovation of resource management are of the most important objectives of sustainable agriculture.

Chemical free traditional farming technologies viz., bio-fertilizers, biodynamics, agnihotra (homa farming), panchagavya, Amrit Pani, rishi krishi, jeevamrutha etc., are gaining a new momentum not only in India, but also world over (Singh et al., 2007) $)^{9}$. These systems offer a means to address self- reliance, rural upliftment and conservation of natural resources. The connections between fungi and the roots of higher plants are referred to as mycorrhiza. Such interactions are mutualistic relationships undertaken by more than 80 per cent of plant species and approximately 6000 species of fungi. Arbuscular mycorrhizal (AM) fungi enable the host plant to establish and grow more efficiently under biotic and abiotic stress conditions, including drought through a series of complex communications between the host and fungus (Salam et al., 2017) ${ }^{7}$. Keeping this in view the present investigation was undertaken to study the Effect of VA Mycorrhizal fungus and bioformulations on quality, root parameters, beneficial microbial count and economics of fennel under northern dry zone (Zone-3) of Karnataka.

\section{Materials and Methods}

The experiment was laid out in sandy loam soil with $\mathrm{pH}$ 6.5-8. Arabhavi is considered to have the benefit of both South-West and North-East monsoon. The mean annual rainfall for the past 20 years of this area is $449.25 \mathrm{~mm}$, distributed over a period of six to seven months from April to November. The monthly mean maximum temperature goes up to $38.45^{\circ} \mathrm{C}$ (March) and monthly mean minimum temperature drops down to $11.55^{\circ} \mathrm{C}$ in January.

The meteorological data for the period of experimentation (Rabi 2017 and 2018) was recorded at the meteorological observatory of the Agricultural Research Station, Arabhavi (UAS, Dharwad) which is situated at $2 \mathrm{~km}$ from the college campus. The experiment was laid out in RCB Design replicated three times using Ajmeer Fennel-1 variety with nine treatments viz., $\mathrm{T}_{1}$-90: 40: $30 \mathrm{~N}: \mathrm{P}: \mathrm{K} \mathrm{kg} \mathrm{ha}^{-1}$ (Check), $\mathrm{T}_{2}-$ VAM $25 \mathrm{~kg} \mathrm{ha}^{-1}, \mathrm{~T}_{3}-$ 90: 40: 30 $\mathrm{N}: \mathrm{P}: \mathrm{K} \mathrm{kg} \mathrm{ha}^{-1}+\mathrm{VAM} 25 \mathrm{~kg} \mathrm{ha}^{-1}, \mathrm{~T}_{4}-\mathrm{VAM}$ $25 \mathrm{~kg} \mathrm{ha}^{-1}+$ Panchagavya 3\% (Drench), $\mathrm{T}_{5-}$ 
VAM $25 \mathrm{~kg} \mathrm{ha}^{-1}+$ Amrutpani 3\% (Drench), $\mathrm{T}_{6}-$ VAM $25 \mathrm{~kg} \mathrm{ha}^{-1}+$ Panchagavya $3 \%+$ Amrutpani 3\% (Drench), T 7 -90: 40: $30 \mathrm{~N}: \mathrm{P}$ : $\mathrm{K} \mathrm{kg} \mathrm{ha}^{-1}+$ Vermiwash $10 \%$ (Drench), $\mathrm{T}_{8}$ VAM $25 \mathrm{~kg} \mathrm{ha}^{-1}+$ Vermiwash 10\% (Drench), $\mathrm{T}_{9}-$ VAM $25 \mathrm{~kg} \mathrm{ha}^{-1}+$ Panchagavya $3 \%+$ Amrutpani 3\% + Vermiwash 10\% (Drench) + Mulch (Sugar cane trash). A spacing of $50 \mathrm{~cm}$ between rows and $25 \mathrm{~cm}$ between the plants was followed. Glomus fasciculatum, a VA Mycorrhizal fungus was applied in the rows of nursery beds prior to sowing of seeds at one $\mathrm{kg} /$ plot. Five representative samples were selected at random from each plot for recording the observations. The average from these five samples were worked out for the statistical computation.

The data recorded for various observations on three experiments were subjected to statistical analysis using the Fischer's method of analysis of variance with 5 per cent level of significance for ' $F$ ' and ' $t$ ' tests. Pooled analysis of 2017 and 2018 was also carried out to draw conclusion. Wherever the ' $\mathrm{F}$ ' test was significant, the critical difference (C.D.) values were worked out at 5 per cent level of significance (Panse and Sukhatme, 1985) (Table 1-5).

\section{Results and Discussion}

\section{Qualitative parameters}

Significant difference was observed for qualitative parameters by the application of VAM and bioformulations. The highest essential oil content (1.04, 1.05 and 1.05\%) was recorded in the treatment $\mathrm{T}_{9}$ (VAM $25 \mathrm{~kg}$ ha $^{-1}+$ Panchagavya 3\% + Amrutpani 3\% + Vermiwash $10 \%$ (Drench) + Mulch (Sugar cane trash). And the lowest $(1.00,1.00$ and $1.00 \%$ ) was recorded in treatment $\mathrm{T}_{2}$ (VAM $25 \mathrm{~kg} \mathrm{ha}^{-1}$ ) in 2017, 2018 and pooled data respectively. The maximum anethole content (59.22, 58.92 and 59.07\%) was recorded in the treatment $\mathrm{T}_{5}$ (VAM $25 \mathrm{~kg} \mathrm{ha}+$ Amrutpani 3\% (Drench)) in 2017, 2018 and pooled data respectively. And the minimum (39.83, 39.56 and $39.70 \%)$ was recorded in treatment $\mathrm{T}_{7}$ (90: 40: $30 \mathrm{~N}: \mathrm{P}: \mathrm{K} \mathrm{kg} \mathrm{ha}^{-1}+$ Vermiwash $10 \%$ (Drench). Findings are in confirmation with Hannah et al., (2005) who reported that banana sprayed with panchagavya solution at 3 per cent resulted in improvement in quality of fruits viz., total soluble sugars, total sugars and reduced the negative quality characters like acidity and ascorbic acid content.

The improvement in quality of fennel with application of VAM and bioformulations may be attributed to the enhanced metabolic activities. Krishnamurthy and Sharanappa $(2005)^{3}$ also reported improved quality parameters of rose onion bulbs through different source of organic nutrient application.

\section{Root parameters}

Significant difference was observed for root attributes by the application of VAM and bioformulations. The highest length of longest root $(26.50,26.25$ and $26.38 \mathrm{~cm})$, was recorded in the treatment $\mathrm{T}_{9}$ (VAM $25 \mathrm{~kg} \mathrm{ha}^{-1}$ + Panchagavya 3\% + Amrutpani 3\% + Vermiwash 10\% (Drench) + Mulch (Sugar cane trash) in 2017, 2018 and pooled data respectively. And the lowest $(21.00,21.00$ and $21.00 \mathrm{~cm}$ ) was recorded in treatment $\mathrm{T}_{7}$ (90: 40: $30 \mathrm{~N}: \mathrm{P}: \mathrm{K} \mathrm{kg} \mathrm{ha}^{-1}+$ Vermiwash $10 \%$ (Drench) which was at par with $\mathrm{T}_{1}(22.00$, 21.50 and $21.75 \mathrm{~cm})$. The highest number of roots $(8.00,8.00$ and 8.00$)$ was recorded in the treatment $\mathrm{T}_{9}$ (VAM $25 \mathrm{~kg} \mathrm{ha}^{-1}+$ Panchagavya $3 \%+$ Amrutpani $3 \%+$ Vermiwash $10 \%$ (Drench) + Mulch (Sugar cane trash) in 2017, 2018 and pooled data respectively. And the lowest (5.00, 4.50 and 4.75) was recorded in treatment $T_{1}$ (90: 40: 30 $\mathrm{N}$ : P: K kg ha ${ }^{-1}$ (Check). 
Table.1 Essential oil in seeds and anethole in oil content as influenced by the application of VAM and bioformulations during rabi 2017 and 2018

\begin{tabular}{|c|c|c|c|c|c|c|}
\hline \multirow[t]{2}{*}{ Treatments } & \multicolumn{3}{|c|}{ Essential oil (\%) } & \multicolumn{3}{|c|}{ Anethole content (\%) } \\
\hline & 2017 & 2018 & Pooled & 2017 & 2018 & Pooled \\
\hline $\mathrm{T}_{1^{-}}$90: 40: $30 \mathrm{~N}: \mathrm{P}: \mathrm{K} \mathrm{kg} \mathrm{ha}^{-1}$ (Check) & 1.01 & 1.01 & 1.01 & 41.06 & 41.03 & 41.05 \\
\hline 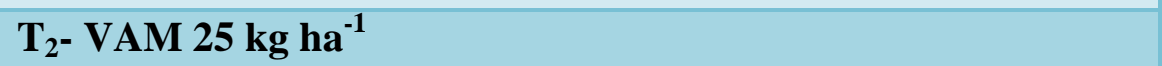 & 1.00 & 1.00 & 1.00 & 44.67 & 44.50 & 44.59 \\
\hline $\mathrm{T}_{3^{-}}$90: 40: $30 \mathrm{~N}: \mathrm{P}: \mathrm{K} \mathrm{kg} \mathrm{ha}^{-1}+$ VAM $25 \mathrm{~kg} \mathrm{ha}^{-1}$ & 1.03 & 1.04 & 1.04 & 56.89 & 56.80 & 56.85 \\
\hline T $_{4}$ - VAM $25 \mathrm{~kg} \mathrm{ha}^{-1}+$ Panchagavya 3\% (Drench) & 1.01 & 1.01 & 1.01 & 44.09 & 44.01 & 44.05 \\
\hline T $_{5}$ - VAM $25 \mathrm{~kg} \mathrm{ha}^{-1}+$ Amrutpani 3\% (Drench) & 1.02 & 1.02 & 1.02 & 59.22 & 58.92 & 59.07 \\
\hline $\begin{array}{l}\text { T }_{6^{-}} \text {VAM } 25 \mathrm{~kg} \mathrm{ha}^{-1}+\text { Panchagavya } 3 \%+\text { Amrutpani } 3 \% \\
\text { (Drench) }\end{array}$ & 1.03 & 1.05 & 1.04 & 49.75 & 49.15 & 49.45 \\
\hline $\mathrm{T}_{7}$ 90: 40: $30 \mathrm{~N}: \mathrm{P}: \mathrm{K} \mathrm{kg} \mathrm{ha}^{-1}+$ Vermiwash $10 \%$ (Drench) & 1.02 & 1.02 & 1.02 & 39.83 & 39.56 & 39.70 \\
\hline T $_{8}$ - VAM $25 \mathrm{~kg} \mathrm{ha}^{-1}+$ Vermiwash $10 \%$ (Drench) & 1.01 & 1.01 & 1.01 & 45.37 & 45.24 & 45.31 \\
\hline 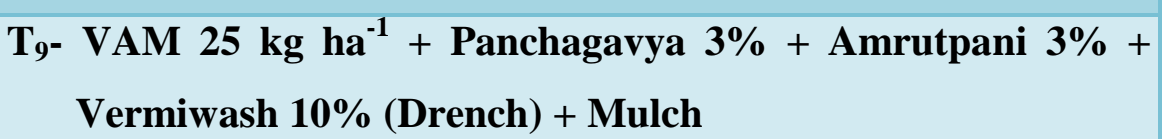 & 1.04 & 1.05 & 1.05 & 49.66 & 49.60 & 49.63 \\
\hline S.Em. \pm & 0.00 & 0.00 & 0.00 & 0.85 & 0.77 & 0.80 \\
\hline CD at $5 \%$ & 0.00 & 0.00 & 0.00 & 2.54 & 2.29 & 2.41 \\
\hline CV (\%) & 12.54 & 12.61 & 12.57 & 14.56 & 13.70 & 14.03 \\
\hline
\end{tabular}

Note: 1. Bioformulations viz., Panchagavya, amrutpani and vermiwash were drenched at monthly interval from the day of sowing up to $2^{\text {nd }}$ harvest

2. Common dose of FYM (12.5 $\left.\mathrm{tha}^{-1}\right)$ was applied at the time of sowing 
Table.2 Per cent root colonization, number of chlamydospores and length of longest root in fennel as influenced by the application of VAM and bioformulations during rabi 2017 and 2018

\begin{tabular}{|c|c|c|c|c|c|c|c|c|c|}
\hline \multirow[t]{2}{*}{ Treatments } & \multicolumn{3}{|c|}{ Per cent root colonization } & \multicolumn{3}{|c|}{$\begin{array}{c}\text { Number of chlamydospores/ } 50 \mathrm{~g} \\
\text { of soil }\end{array}$} & \multicolumn{3}{|c|}{ Length of longest root $(\mathrm{cm})$} \\
\hline & 2017 & 2018 & Pooled & 2017 & 2018 & Pooled & 2017 & 2018 & Pooled \\
\hline $\begin{array}{l}\text { T}_{1-} \text { 90: 40: } 30 \mathrm{~N}: \mathrm{P}: \mathrm{K} \mathrm{kg} \mathrm{ha}^{-1} \\
\text { (Check) }\end{array}$ & 40.00 & 41.00 & 40.50 & 325.00 & 299.00 & 312.00 & 22.00 & 21.50 & 21.75 \\
\hline T $_{2}-$ VAM $25 \mathrm{~kg} \mathrm{ha}^{-1}$ & 81.00 & 79.00 & 80.00 & 1265.00 & 1164.00 & 1214.50 & 23.00 & 22.80 & 22.90 \\
\hline $\begin{array}{l}T_{3-} \text { 90: } 40: 30 \mathrm{~N}: \text { P: } \mathrm{K} \mathrm{kg} \mathrm{ha}^{-1}+ \\
\text { VAM } 25 \mathrm{~kg} \mathrm{ha}^{-1}\end{array}$ & 94.00 & 92.00 & 93.00 & 1140.00 & 1049.00 & 1094.50 & 24.00 & 23.00 & 23.50 \\
\hline 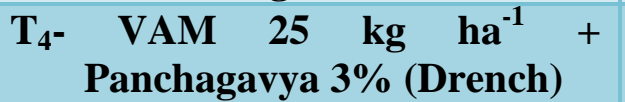 & 86.00 & 86.00 & 86.00 & 1290.00 & 1187.00 & 1238.50 & 24.00 & 24.00 & 24.00 \\
\hline T $_{5^{-}} \underset{\text { Amrutpani 3\% }}{\text { VAM }} \underset{\text { (Drench) }}{25} \quad \mathrm{~kg}_{\text {(Drech }}^{\mathrm{ha}^{-1}}+$ & 91.00 & 91.00 & 91.00 & 1325.00 & 1219.00 & 1272.00 & 23.70 & 23.25 & 23.48 \\
\hline $\begin{array}{lccc}\text { T}_{6^{-}} & \underset{\text { VAM }}{\text { VAM }} \mathbf{2 5} & \mathrm{ka}^{-1} & + \\
\text { Panchagavya } & 3 \% & + \\
\text { Amrutpani 3\% } & \text { (Drench) } & \end{array}$ & 94.00 & 93.00 & 93.50 & 1370.00 & 1260.00 & 1315.00 & 25.40 & 25.00 & 25.20 \\
\hline $\begin{array}{l}\mathrm{T}_{7} \text { - 90: } 40: 30 \mathrm{~N}: \mathrm{P}: \mathrm{K} \mathrm{kg} \mathrm{ha}^{-1}+ \\
\text { Vermiwash } 10 \% \text { (Drench) }\end{array}$ & 89.00 & 89.00 & 89.00 & 550.00 & 506.00 & 528.00 & 21.00 & 21.00 & 21.00 \\
\hline T $_{8^{-}} \underset{\text { Vermiwash }}{\text { VAM }} \underset{10 \%}{25} \mathrm{~kg}_{(\text {Drench) }}^{\mathrm{ha}^{-1}}+$ & 85.00 & 85.00 & 85.00 & 1210.00 & 1113.00 & 1161.50 & 25.10 & 25.00 & 25.05 \\
\hline $\begin{array}{l}\text { T9- }_{9}^{\text {VAM } 25} \text { kg } \text { ha }^{-1}+ \\
\text { Panchagavya } \\
\text { Amrutpani } 3 \% \\
\text { A\% + Vermiwash } \\
\text { 10\% (Drench) }+ \text { Mulch }\end{array}$ & 96.00 & 95.00 & 95.50 & 1430.00 & 1316.00 & 1373.00 & 26.50 & 26.25 & 26.38 \\
\hline S.Em. \pm & 2.15 & 1.55 & 1.40 & 44.75 & 35.62 & 40.00 & 0.44 & 0.44 & 0.44 \\
\hline CD at $5 \%$ & 6.44 & 4.64 & 4.18 & 134.17 & 106.78 & 119.93 & 1.32 & 1.32 & 1.32 \\
\hline CV $(\%)$ & 19.99 & 19.97 & 19.68 & 14.62 & 14.79 & 14.68 & 15.24 & 15.30 & 15.26 \\
\hline
\end{tabular}

Note: 1. Bioformulations viz., Panchagavya, amrutpani and vermiwash were drenched at monthly interval from the day of sowing up to $2^{\text {nd }}$ harvest 2. Common dose of FYM $\left(12.5 \mathrm{tha}^{-1}\right)$ was applied at the time of sowing 
Table.3 Total number of roots, root volume and bacterial count in fennel at harvest as influenced by the application of VAM and bioformulations during rabi 2017 and 2018

\begin{tabular}{|c|c|c|c|c|c|c|c|c|c|}
\hline \multirow[t]{2}{*}{ Treatments } & \multicolumn{3}{|c|}{ Total number of roots } & \multicolumn{3}{|c|}{ Root volume $\left(\mathrm{cm}^{3}\right)$} & \multicolumn{3}{|c|}{$\begin{array}{c}\text { Bacteria No. } \times 10^{6} \mathrm{CFU} / \mathrm{g} \text { of } \\
\text { soil }\end{array}$} \\
\hline & 2017 & 2018 & Pooled & 2017 & 2018 & Pooled & 2017 & 2018 & Pooled \\
\hline $\begin{array}{l}\text { T}_{1-} \text { 90: 40: } 30 \mathrm{~N}: \mathrm{P}: \mathrm{K} \mathrm{kg} \mathrm{ha}^{-1} \\
\text { (Check) }\end{array}$ & 5.00 & 4.50 & 4.75 & 40.00 & 38.00 & 39.00 & 23.00 & 21.00 & 22.00 \\
\hline $\mathrm{T}_{2^{-}}$VAM $25 \mathrm{~kg} \mathrm{ha}^{-1}$ & 6.50 & 6.00 & 6.25 & 55.00 & 52.00 & 53.50 & 106.00 & 99.00 & 102.50 \\
\hline $\begin{array}{l}\text { T}_{3^{-}} \text {90: 40: } 30 \mathrm{~N}: \mathrm{P}: \mathrm{K} \mathrm{kg} \mathrm{ha}^{-1}+\mathrm{VAM} \\
25 \mathrm{~kg} \mathrm{ha}^{-1}\end{array}$ & 7.00 & 7.00 & 7.00 & 65.00 & 66.00 & 65.50 & 97.00 & 90.00 & 93.50 \\
\hline $\begin{array}{l}\text { T}_{4}-\text { VAM } 25 \mathrm{~kg} \mathrm{ha}^{-1}+\text { Panchagavya } \\
\text { 3\% (Drench) }\end{array}$ & 6.00 & 5.67 & 5.83 & 45.00 & 42.00 & 43.50 & 118.00 & 110.00 & 114.00 \\
\hline $\begin{array}{l}\text { T }_{5-} \\
\text { 3\% VAM } 25 \mathrm{~kg} \mathrm{ha}^{-1}+\text { Amrutpani }\end{array}$ & 7.66 & 7.50 & 7.58 & 63.00 & 65.00 & 64.00 & 123.00 & 114.00 & 118.50 \\
\hline 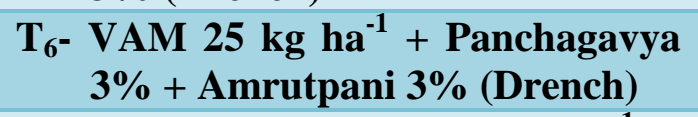 & 7.00 & 7.00 & 7.00 & 68.00 & 69.00 & 68.50 & 135.00 & 126.00 & 130.50 \\
\hline $\begin{array}{l}\mathrm{T}_{7^{-}} \text {90: 40: } 30 \mathrm{~N}: \mathrm{P}: \mathrm{K} \mathrm{kg} \mathrm{ha} \mathrm{h}^{-1}+ \\
\text { Vermiwash 10\% (Drench) }\end{array}$ & 5.00 & 4.50 & 4.75 & 60.00 & 60.00 & 60.00 & 93.00 & 87.00 & 90.00 \\
\hline $\begin{array}{l}\text { T }_{8^{-}} \text {VAM } 25 \mathrm{~kg} \mathrm{ha}^{-1}+\text { Vermiwash } \\
10 \% \text { (Drench) }\end{array}$ & 7.00 & 7.00 & 7.00 & 43.00 & 40.00 & 41.50 & 110.00 & 103.00 & 106.50 \\
\hline 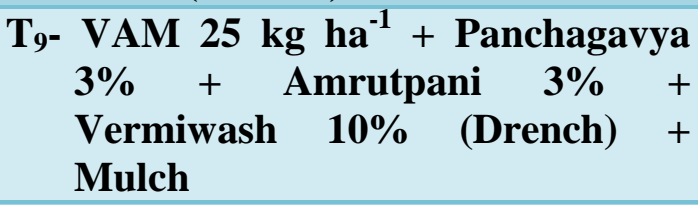 & 8.00 & 8.00 & 8.00 & 70.00 & 69.00 & 69.50 & 144.00 & 134.00 & 139.00 \\
\hline S.Em. \pm & 0.15 & 0.07 & 0.08 & 1.09 & 0.88 & 0.60 & 2.03 & 2.93 & 2.04 \\
\hline CD at $5 \%$ & 0.45 & 0.20 & 0.25 & 3.27 & 2.65 & 1.80 & 6.08 & 8.78 & 6.11 \\
\hline CV (\%) & 16.84 & 19.80 & 18.19 & 19.93 & 18.18 & 18.90 & 13.26 & 12.71 & 12.92 \\
\hline
\end{tabular}

Note: 1. Bioformulations viz., Panchagavya, amrutpani and vermiwash were drenched at monthly interval from the day of sowing up to $2^{\text {nd }}$ harvest

2. Common dose of FYM $\left(12.5 \mathrm{t} \mathrm{ha}^{-1}\right)$ was applied at the time of sowing 
Table.4 Fungi and actinomycetes count in fennel roots as influenced by the application of VAM and bioformulations during rabi 2017 and 2018

\begin{tabular}{|c|c|c|c|c|c|c|}
\hline \multirow[t]{2}{*}{ Treatments } & \multicolumn{3}{|c|}{$\begin{array}{c}\text { Number of fungi } \times 10^{3} \mathrm{CFU} / \mathrm{g} \text { of } \\
\text { soil }\end{array}$} & \multicolumn{3}{|c|}{$\begin{array}{l}\text { Number of actinomycetes } \times \\
\qquad 10^{4} \mathrm{CFU} / \mathrm{g} \text { of soil }\end{array}$} \\
\hline & 2017 & 2018 & Pooled & 2017 & 2018 & Pooled \\
\hline T1- 90: 40: $30 \mathrm{~N}: \mathrm{P}: \mathrm{K} \mathrm{kg} \mathrm{ha}^{-1}$ (Check) & 52.00 & 48.00 & 50.00 & 23.00 & 21.00 & 22.00 \\
\hline $\mathrm{T}_{2}-$ VAM $25 \mathrm{~kg} \mathrm{ha}^{-1}$ & 169.00 & 157.00 & 163.00 & 59.00 & 55.00 & 57.00 \\
\hline T3- 90: 40: $30 \mathrm{~N}:$ P: $\mathrm{K} \mathrm{kg} \mathrm{ha}^{-1}+$ VAM $25 \mathrm{~kg} \mathrm{ha}^{-1}$ & 154.00 & 143.00 & 148.50 & 54.00 & 50.00 & 52.00 \\
\hline T$_{4}$ - VAM $25 \mathrm{~kg} \mathrm{ha}^{-1}+$ Panchagavya $3 \%$ (Drench) & 207.00 & 193.00 & 200.00 & 71.00 & 66.00 & 68.50 \\
\hline T $_{5}$ - VAM $25 \mathrm{~kg} \mathrm{ha}^{-1}+$ Amrutpani 3\% (Drench) & 221.00 & 206.00 & 213.50 & 76.00 & 71.00 & 73.50 \\
\hline $\begin{array}{l}\text { T }_{6-} \text { VAM } 25 \mathrm{~kg} \mathrm{ha}^{-1}+\text { Panchagavya 3\% + Amrutpani 3\% } \\
\text { (Drench) }\end{array}$ & 234.00 & 218.00 & 226.00 & 81.00 & 75.00 & 78.00 \\
\hline $\mathrm{T}_{7^{-}}$90: 40: $30 \mathrm{~N}: \mathrm{P}: \mathrm{K} \mathrm{kg} \mathrm{ha}^{-1}+$ Vermiwash 10\% (Drench) & 151.00 & 140.00 & 145.50 & 47.00 & 44.00 & 45.50 \\
\hline 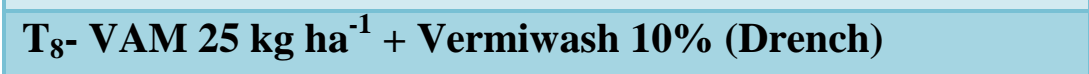 & 186.00 & 173.00 & 179.50 & 63.00 & 59.00 & 61.00 \\
\hline 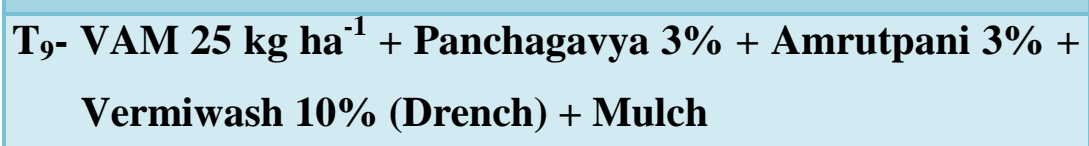 & 242.00 & 225.00 & 233.50 & 84.00 & 78.00 & 81.00 \\
\hline S.Em. \pm & 3.37 & 4.55 & 3.19 & 2.15 & 1.59 & 1.37 \\
\hline CD at $5 \%$ & 10.10 & 13.65 & 9.56 & 6.44 & 4.77 & 4.11 \\
\hline CV $(\%)$ & 11.37 & 13.36 & 12.75 & 11.21 & 11.39 & 11.21 \\
\hline
\end{tabular}

Note: 1. Bioformulations viz., Panchagavya, amrutpani and vermiwash were drenched at monthly interval from the day of sowing up to $2^{\text {nd }}$ harvest

2. Common dose of FYM (12.5 tha $\left.\mathrm{ta}^{-1}\right)$ was applied at the time of sowing 
Table.5 Effect of VAM and bioformulations on economics of Fennel

\begin{tabular}{|c|c|c|c|c|c|c|c|c|c|c|c|c|}
\hline \multirow[t]{3}{*}{ Treatments } & \multicolumn{12}{|c|}{ Economics (C) } \\
\hline & \multicolumn{4}{|c|}{2017} & \multicolumn{4}{|c|}{2018} & \multicolumn{4}{|c|}{ Pooled } \\
\hline & $\begin{array}{l}\text { Total } \\
\text { Cost }\end{array}$ & $\begin{array}{c}\text { Gross } \\
\text { returns }\end{array}$ & $\begin{array}{c}\text { Net } \\
\text { returns }\end{array}$ & $\begin{array}{l}\mathrm{B}: \mathrm{C} \\
\text { ratio }\end{array}$ & $\begin{array}{l}\text { Total } \\
\text { Cost }\end{array}$ & $\begin{array}{c}\text { Gross } \\
\text { returns }\end{array}$ & $\begin{array}{c}\text { Net } \\
\text { returns }\end{array}$ & $\begin{array}{l}\mathrm{B}: \mathrm{C} \\
\text { ratio }\end{array}$ & $\begin{array}{l}\text { Total } \\
\text { Cost }\end{array}$ & $\begin{array}{l}\text { Gross } \\
\text { returns }\end{array}$ & $\begin{array}{c}\text { Net } \\
\text { returns }\end{array}$ & $\begin{array}{l}\text { B:C } \\
\text { ratio }\end{array}$ \\
\hline $\mathrm{T}_{1}$ & 39312 & 139620 & 100308 & 3.55 & 39312 & 134030 & 94718 & 3.41 & 39312 & 136825 & 97513 & 3.48 \\
\hline $\mathrm{T}_{2}$ & 40600 & 130260 & 89660 & 3.21 & 40600 & 124930 & 84330 & 3.08 & 40600 & 127595 & 86995 & 3.14 \\
\hline $\mathrm{T}_{3}$ & 41812 & 151840 & 110028 & 3.63 & 41812 & 145730 & 103918 & 3.49 & 41812 & 148785 & 106973 & 3.56 \\
\hline $\mathrm{T}_{4}$ & 41350 & 133250 & 91900 & 3.22 & 41350 & 127920 & 86570 & 3.09 & 41350 & 130585 & 89235 & 3.15 \\
\hline $\mathrm{T}_{5}$ & 41200 & 145860 & 104660 & 3.54 & 41200 & 140010 & 98810 & 3.40 & 41200 & 142935 & 101735 & 3.47 \\
\hline $\mathrm{T}_{6}$ & 41950 & 157690 & 115740 & 3.76 & 41950 & 151320 & 109370 & 3.61 & 41950 & 154505 & 112555 & 3.68 \\
\hline $\mathrm{T}_{7}$ & 40562 & 142610 & 102048 & 3.52 & 40562 & 136890 & 96328 & 3.37 & 40562 & 139750 & 99188 & 3.44 \\
\hline $\mathrm{T}_{8}$ & 41850 & 132340 & 90490 & 3.16 & 41850 & 127010 & 85160 & 3.03 & 41850 & 129675 & 87825 & 3.09 \\
\hline $\mathrm{T}_{9}$ & 43200 & 162500 & 119300 & 3.76 & 43200 & 156000 & 112800 & 3.61 & 43200 & 159250 & 116050 & 3.68 \\
\hline \multicolumn{13}{|c|}{ 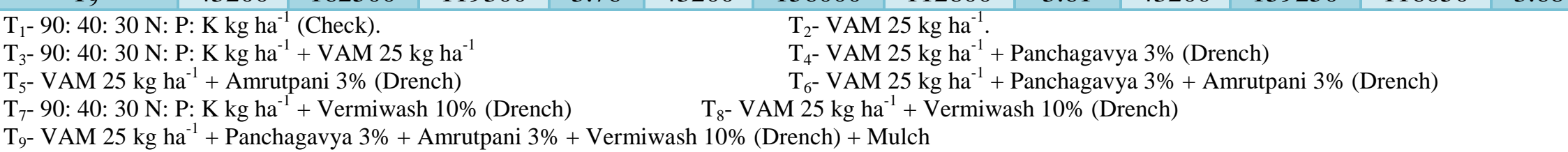 } \\
\hline
\end{tabular}


Significant difference was observed for root volume by the application of VAM and bioformulations. The highest root volume $\left(70.00,69.00\right.$ and $\left.69.50 \mathrm{~cm}^{-3}\right)$ was recorded in the treatment $\mathrm{T}_{9}$ (VAM $25 \mathrm{~kg} \mathrm{ha}^{-1}+$ Panchagavya 3\%+ Amrutpani 3\%+ Vermiwash 10\% (Drench) + Mulch (Sugar cane trash) which was at par with $\mathrm{T}_{6}(68.00$, 69.00 and $69.50 \mathrm{~cm}^{3}$ ) in 2017, 2018 and pooled data respectively. And the lowest $\left(40.00,38.00\right.$ and $\left.39.00 \mathrm{~cm}^{3}\right)$ was recorded in treatment $\mathrm{T}_{1}\left(90: 40: 30 \mathrm{~N}: \mathrm{P}: \mathrm{K} \mathrm{kg} \mathrm{ha}^{-1}\right.$ (Check).

\section{Microbial analysis}

The highest per cent root colonization (96.00, 95.00 and $95.50 \%$ ) was recorded in the

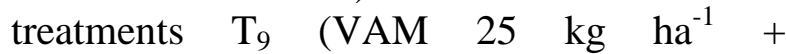
Panchagavya 3\%+ Amrutpani 3\%+ Vermiwash 10\% (Drench) + Mulch (Sugar cane trash) which was at par with $\mathrm{T}_{6}(94.00$, 93.00 and $93.50 \%), \mathrm{T}_{3}(94.00,92.00$ and $93.00 \%)$ and $\mathrm{T}_{5}(91.00,91.00$ and $91.00 \%)$ in 2017, 2018 and pooled data respectively. And the lowest $(40.00,41.00$ and $40.50 \%)$ was recorded in treatment $\mathrm{T}_{1}(90: 40: 30 \mathrm{~N}: \mathrm{P}: \mathrm{K}$ $\mathrm{kg} \mathrm{ha}{ }^{-1}$ (Check). The highest number of chlamydospores per $50 \mathrm{~g}$ of soil (1430.00, 1316.00 and 1373.00) was recorded in the treatments $\mathrm{T}_{9} \quad$ (VAM $25 \quad \mathrm{~kg} \quad \mathrm{ha}^{-1}+$ Panchagavya 3\%+ Amrutpani 3\%+ Vermiwash 10\% (Drench) + Mulch (Sugar cane trash) which was at par with $\mathrm{T}_{6}(1370.00$, 1260.00 and 1315.00) and $\mathrm{T}_{5}$ (1325.00, 1219.00 and 1272.00) in 2017, 2018 and pooled data respectively. And the lowest (325.00, 299.00 and 312.00) was recorded in treatment $\mathrm{T}_{1}$ (90: 40: $30 \mathrm{~N}: \mathrm{P}: \mathrm{K} \mathrm{kg} \mathrm{ha}^{-1}$ (Check).

\section{Beneficial microbial count}

Significant difference was observed for bacterial count per gram of soil by the application of VAM and bioformulations. The highest bacterial count (144.00, 134.00 and $139.00 \times 10^{6} \mathrm{CFU} / \mathrm{g}$ of soil) was recorded in the treatment $\mathrm{T}_{9}$ (VAM $25 \mathrm{~kg} \mathrm{ha}{ }^{-1}+$ Panchagavya 3\%+ Amrutpani 3\%+ Vermiwash 10\% (Drench) + Mulch (Sugar cane trash) in 2017, 2018 and pooled data respectively. And the lowest $(23.00,21.00$ and $22.00 \times 10^{6} \mathrm{CFU} / \mathrm{g}$ of soil) was recorded in treatment $\mathrm{T}_{1}$ (90: 40: $30 \mathrm{~N}: \mathrm{P}: \mathrm{K} \mathrm{kg} \mathrm{ha}^{-1}$ (Check). The highest fungal count (242.00, 225.00 and $233.50 \times 10^{3} \mathrm{CFU} / \mathrm{g}$ of soil) was recorded in the treatment $T_{9}$ (VAM $25 \mathrm{~kg} \mathrm{ha}^{-1}$ + Panchagavya 3\% + Amrutpani 3\% + Vermiwash 10\% (Drench) + Mulch (Sugar cane trash) which was at par with $\mathrm{T}_{6}(234.00$, 218.00 and $226.00 \times 10^{3} \mathrm{CFU} / \mathrm{g}$ of soil) in 2017, 2018 and pooled data respectively. And the lowest $\left(52.00,48.00\right.$ and $50.00 \times 10^{6}$ $\mathrm{CFU} / \mathrm{g}$ of soil) was recorded in treatment $\mathrm{T}_{1}$ (90: 40: $30 \mathrm{~N}$ : P: $\mathrm{K} \mathrm{kg} \mathrm{ha}^{-1}$ (Check). The highest actinomycetes count $(84.00,78.00$ and $81.00 \times 10^{4} \mathrm{CFU} / \mathrm{g}$ of soil) was recorded in the treatment $\mathrm{T}_{9}$ (VAM $25 \mathrm{~kg} \mathrm{ha}^{-1}+$ Panchagavya $3 \%+$ Amrutpani $3 \%+$ Vermiwash 10\% (Drench) + Mulch (Sugar cane trash) which was at par with $\mathrm{T}_{6}$ $\left(81.00,75.00\right.$ and $78.00 \times 10^{6} \mathrm{CFU} / \mathrm{g}$ of soil) in 2017, 2018 and pooled data respectively. And the lowest $\left(23.00,21.00\right.$ and $22.00 \times 10^{4}$ $\mathrm{CFU} / \mathrm{g}$ of soil) was recorded in treatment $\mathrm{T}_{1}$ (90: 40: $30 \mathrm{~N}$ : P: $\mathrm{K} \mathrm{kg} \mathrm{ha}^{-1}$ (Check).

\section{Economics analysis}

Higher cost of production (43200, 43200 and $43200 \mathrm{Rs} / \mathrm{ha}$ ) was accounted in the treatment $\mathrm{T}_{9}$ (VAM $25 \mathrm{~kg} \mathrm{ha}^{-1}+$ Panchagavya $3 \%+$ Amrutpani 3\% + Vermiwash 10\% (Drench) + Mulch (Sugar cane trash) in 2017, 2018 and pooled data respectively. While the lower (39312, 39312 and 39312 Rs/ha) was accounted in treatment $T_{1}(90: 40: 30 \mathrm{~N}: \mathrm{P}: \mathrm{K} \mathrm{kg}$ $\left.\mathrm{ha}^{-1}\right)$. Highest gross returns $(162500,156000$ and $159250 \mathrm{Rs} / \mathrm{ha})$, net returns (119300, 112800 and $116050 \mathrm{Rs} / \mathrm{ha}$ ) and Benefit:cost ratio (3.76, 3.61 and 3.69) were accounted in 
the treatment $\mathrm{T}_{9}$ (VAM $25 \mathrm{~kg} \mathrm{ha}^{-1}+$ Panchagavya 3\%+ Amrutpani 3\%+ Vermiwash $10 \%$ (Drench) + Mulch (Sugar cane trash) in 2017, 2018 and pooled data respectively. While the lowest (130260, 124930 and $127595 \mathrm{Rs} / \mathrm{ha}),(89660,84330$ and $86995 \mathrm{Rs} / \mathrm{ha}$ ) and (3.16, 3.03 and 3.10) were accounted in treatment $T_{1}$ (90:40:30 $\mathrm{N}: \mathrm{P}: \mathrm{K} \mathrm{kg} \mathrm{ha}^{-1}$ ) and $\mathrm{T}_{8}$ (VAM $25 \mathrm{~kg} \mathrm{ha}^{-1}+$ Vermiwash $10 \%$ (Drench) respectively. Similar findigs were also reported by Meena et al., (2013) ${ }^{4}$, Rana et al., (2015) ${ }^{6}$ and Singh and Amin (2015) ${ }^{8}$ (Table 5).

Significantly higher essential oil content (1.04, 1.05 and $1.05 \%)$ was recorded in the treatment $\mathrm{T}_{9}$ (VAM $25 \mathrm{~kg} \mathrm{ha}^{-1}+$ Panchagavya $3 \%+$ Amrutpani 3\% + Vermiwash 10\% (Drench) + Mulch (Sugar cane trash) in 2017, 2018 and pooled data respectively.

Significantly higher anethole content (59.22, 58.92 and $59.07 \%$ ) was recorded in the treatment $\mathrm{T}_{5}$ (VAM $25 \mathrm{~kg} \mathrm{ha}^{-1}+$ Amrutpani 3\% (Drench)) in 2017, 2018 and in the pooled data respectively.

Significantly higher per cent root colonization (96.00, 95.00 and 95.50), number of chlamydospores per $50 \mathrm{~g}$ of soil (1430.00, 1316.00 and 1373.00), length of longest root $(26.50,26.25$ and $26.38 \mathrm{~cm})$, number of roots (8.00, 8.00 and 8.00), root volume (70.00, 69.00 and $\left.69.50 \mathrm{~cm}^{3}\right)$, bacterial count $(144.00$, 134.00 and $139.00 \times 10^{6} \mathrm{CFU} / \mathrm{g}$ of soil), fungal count $(242.00,225.00$ and $233.50 \times$ $10^{3} \mathrm{CFU} / \mathrm{g}$ of soil) and actinomycetes count $\left(84.00,78.00\right.$ and $81.00 \times 10^{4} \mathrm{CFU} / \mathrm{g}$ of soil) were recorded in the treatment $\mathrm{T}_{9}$ (VAM 25 $\mathrm{kg} \mathrm{ha}^{-1}+$ Panchagavya 3\% + Amrutpani 3\% + Vermiwash $10 \%$ (Drench) + Mulch (Sugar cane trash) in 2017, 2018 and in the pooled data respectively.

Maximum net returns (119300, 112800 and $116050 \mathrm{Rs} / \mathrm{ha}$ ) and Benefit:cost ratio (3.76,
3.61 and 3.69) were accounted in the treatment $\mathrm{T}_{9}$ (VAM $25 \mathrm{~kg} \mathrm{ha}^{-1}+$ Panchagavya $3 \%+$ Amrutpani 3\% + Vermiwash 10\% (Drench) + Mulch (Sugar cane trash) in 2017, 2018 and in the pooled data respectively.

In conclusion the application of VAM and bio-formulations along with optimum dose of fertilizer influenced the quality attributes, root parameters, beneficial microbial count and economics of fennel. Application of VAM (25 $\mathrm{kg} \mathrm{ha}{ }^{-1}$ ) along with foliar spray of Panchagavya (3\%), Amrutpani (3\%) + Vermiwash 10\% (Drench) + Mulch (Sugar cane trash) resulted in higher values for quality attributes, root parameters, beneficial microbial count and economics of fennel under northern dry zone of Karnataka.

\section{References}

Brown, M. E. 1972. Plant growth substances produced by microorganisms of soil and rhizosphere. J. Appl. Bact. 35: 443.

Kale, R. N., Bano, K. and Satyavati, G. P. 1991. Influence of vermicompost application on growth and yield of cereals, vegetables and ornamental plants. Final Report of KSCSI Project. 34B. 34-78.

Krishnamurthy, D. and Sharanappa. 2005. Effect of sole and integrated use of improved composts and NPK fertilizers on the quality, productivity and shelf life of Bangalore Rose Red onion (Alium cepa L.). Mysore J. Agril. Sci. 39(2): 355-361.

Meena, S. S., Mehta, R. S., Meena, R. D., Meena, N. K. and Singh, B. 2013. Effect of sowing time and crop geometry on growth and seed yield of dill (Anethum sowa L.) Int. J. Seed Spices. 3(2): 81-84.

Panse, U. G. and Sukhatme, B. V. 1985. Statistical methods for Agricultural 
workers. Indian Council of Agricultural Research, New Delhi. pp. 100-161.

Rana, S. C., Pandita, V. K. and Sanjai, S. 2015. Influence of spacing and number of leaf cutting on seed yield in fenugreek. Legume Res. 38(6): 858:860.

Salam, E. A., Alatar, A. and El-Sheikh, M. A. 2017. Inoculation with arbuscular mycorrhizal fungi alleviates harmful effects of drought stress on damask rose. Saudi J. Biol. Sci. 9(3): 44-49.
Singh, A. and Amin, A. U. 2015. Response of drilled rabi fennel (Foeniculum vulgare Mill) to spacing under varying levels of nitrogen. International J. Seed Spices. 5(1): 102-104.

Singh, B., Ranjan, S. and Ramchandra. 2007. Response of panchagavya and manchurian mushroom tea on floral characters in tuberose (Polyanthus tuberose Linn.) cv. Pearl Double. J. Ornamental Hort. 10 (4): 250-254.

\section{How to cite this article:}

Shashidhar M. Dodamani, N. K. Hegde, Chaya P. Patil, Laxman Kukanoor, T. B. Alloli and Krishna Kurubetta. 2020. Effect of VA Mycorrhizal Fungus and Bioformulations on Quality, Root Parameters, Microbial Analysis and Economics of Fennel under Northern Dry Zone (ZONE-3) of Karnataka. Int.J.Curr.Microbiol.App.Sci. 9(12): 769-779. doi: https://doi.org/10.20546/ijcmas.2020.912.092 